\title{
Quality of Life in Women with Endometriosis Pelvic Pain Treated with the Levonorgestrel-Releasing Intrauterine System
}

\author{
René Cortés Flores, Evangelina Briones Lara, Luis Carlos Quintana Corral, \\ Ricardo Alberto Isacc Chaib, Luis Oswaldo de la 0. Pérez, Oscar Armando González Díaz, \\ Raúl Cortés Flores
}

Obstetrics and Gynecology Hospital, UMAE 23 “Dr. Ignacio Morones Prieto” Mexican Institute of Social Security, Monterrey, Mexico

Email: evangelina.briones@imss.gob.mx

Received 1 February 2015; accepted 16 March 2015; published 17 March 2015

Copyright (C) 2015 by authors and Scientific Research Publishing Inc.

This work is licensed under the Creative Commons Attribution International License (CC BY). http://creativecommons.org/licenses/by/4.0/

(c) (i) Open Access

\section{Abstract}

Objective: To evaluate the quality of life in patients with endometriosis pelvic pain before and after the application of the levonorgestrel-releasing intrauterine system (LNG-IUS). Design: Open non-comparative study. Setting: Obstetrics and Gynecology Hospital at Monterrey, Mexico. Sample: 29 women aged 18 to 40 years with pelvic pain associated with endometriosis confirmed by laparoscopy. Methods: After laparoscopy but before LNG-IUS insertion (basal visit) and 6 months afterwards, modified Endometriosis Health Profile (EHP-30) was applied. Main outcomes measures: Size of change of questionnaire scores, need of additional analgesic therapy and adverse effects. Statistical Analysis: Differences in the questionnaire scores before and after intervention were analyzed by Student t-test. Results: Final analysis set included 29 women aged $31.7 \pm 4.7$ years years. The ASRM surgical staging of endometriosis was mild in 19.3 moderate in 13.7 and severe in $76 \%$ of the patients. The general perception of quality of life improved from 52 at baseline to $98 \%$ at six months $(p<0.001)$. Adverse events were mild in nature, 19 patients reported no adverse events during the study (65.5\%). Two patients $(6.9 \%)$ required the use of concomitant therapy with non-steroidal analgesics for relief of pain. Conclusion: The application of LNG-IUS in patients with pelvic pain associated with endometriosis improved significatively all aspects related with quality of life as measured with Endometriosis Health Profile (EHP-30). We concluded that LNGIUS may be an effective and convenient therapeutic alternative for the management of pain associated with endometriosis. 


\section{Keywords}

\section{Quality of Life, Endometriosis, Chronic Pelvic Pain, Levonorgestrel-Releasing Intrauterine System, LNG-IUS, EPH-30, Questionnaires}

\section{Introduction}

Endometriosis is a relatively common disorder clinically manifested as chronic pelvic pain and/or infertility. It is characterized by the presence of functional endometrial glands and stroma outside the uterus [1]. Presumably 80 million women are affected by the disease worldwide. It is assumed that the prevalence of endometriosis is up to 15 percent of all women of reproductive age [2]. Endometriosis affects 21 to 47 percent of women with infertility, 71 to 87 percent of those with chronic pelvic pain, and 69 percent of adolescents with pelvic pain refractory to treatment [3] [4]. In Mexico, there is scant epidemiological data on endometriosis; the incidence is reported only in groups of patients with specific ailments as infertility in whom incidence is around $34.5 \%$ [5].

Traditionally, the therapeutic goal in the hormonal treatment of endometriosis has been the suppression of ovarian activity. Accordingly, approved treatments in the management of endometriosis include aGnRH analogues, progestins and danazol. Although oral combined contraceptives have not approved indication, they have been investigated and are widely prescribed for the treatment of endometriosis pelvic pain [6].

The levonorgestrel-releasing intrauterine system (LNG-IUS), besides its contraceptive effect, has a strong effect on the endometrium causing its inactivation and atrophy without suppressing ovulation; this is due to the high hormonal dose locally released that stops endometrial proliferation increasing apoptosis of endometrial glands and stroma. LNG-IUS is a T-shaped flexible structure, which includes a hormonal reservoir in its vertical arm containing $52 \mathrm{mg}$ of levonorgestrel. Hormonal initial release is $20 \mathrm{mcg} /$ day, decreasing progressively to a release of $12 \mathrm{mcg}$ at the end of the 5th year of use [7]. Considering the strong local action on endometrium, some studies have shown the plausibility to use the LNG-IUS as an effective option in the treatment of chronic pelvic pain associated with endometriosis, with few systemic adverse events.

The quality of life related to health is a multidimensional and dynamic concept that encompasses the physical, psychological and social aspects associated with a disease or its treatment [8]. One method to assess the outcome of different treatments for endometriosis is based on the concept of quality of life as the patient's perception of the impact in different areas of her life, especially physical, emotional and social well-being comprised by her disease [9]. The Endometriosis Health Profile (EHP-30) is a Health Related Quality of Life (HRQoL) patient self-report of outcomes (PRO); used to measure the wide range of effects that endometriosis can have on women's lives. The EHP-30 is the only condition-specific PRO designed from the patient's perspective to assess health related quality of life in endometriosis. Published evidence concludes that the EHP-30 is a reliable and valid instrument for assessing areas of concern to women with endometriosis that are not addressed by other condition-specific and generic questionnaires [10] [11]. The core instruments have five scale scores covering: Pain, control and powerlessness, social support, emotional well-being and self-image.

With the aim to evaluate the quality of life in patients with endometriosis pelvic pain before and after the application of the levonorgestrel-releasing intrauterine system (LNG-IUS), we designed this quasi-experimental pre-post test study using The Endometriosis Health Profile (EHP-30) questionnaire.

\section{Material and Methods}

\subsection{Setting}

After approval by the local investigation and ethics committees, this study was conducted from January 1st to December 30th, 2012 at Obstetrics and Gynecology Hospital UMAE 23 “Dr. Ignacio Morones Prieto" Mexican Institute of Social Security, Monterrey, Mexico.

\subsection{Sample}

After screening, twenty-nine women with chronic pelvic pain referred by their family physicians to specialty 
consultation at Obstetrics and Gynecology Hospital, who signed informed consent for participation in the study, were included. The mean age was $31.7 \pm 4.7$ years. Only two patients were referred with suspected diagnosis of endometriosis. Laparoscopy procedures were done in all patients to diagnose, and treat endometriosis. The severity of the disease by ASRM Staging System were mild (Stage II) in three patients (10.3\%); moderate (Stage III) in four patients (13.7\%); and severe (Stage IV) in 22 patients (76\%). Surgical treatment consisting in resection of endometriotic foci and adhesiolysis was performed in 17 patients, Laparoscopic Uterine Nerve Ablation (LUNA) in 7 patients, resection of endometriomas in 2, and no surgical treatment in 3 patients. Main exclusion criteria were: patients on hormone therapy in the 3 months prior to inclusion; patients with contraindications for the use of LNG-IUS or patients whom desire to become pregnant.

The LNG-IUS was applied during the first menstruation after laparoscopy.

\subsection{Methods}

The EHP-30 consists of a Likert scale with five possible responses (never, rarely, sometimes, often, and always). The questionnaire assesses core aspects of pain (9 items), emotional well-being and self-image (11 items), alongside we selected supplementary modules including work (5 items), sexual intercourse (5 items) and perceptions about treatment (5 items). Section of questions in the original questionnaire about fertility was removed because of the nature of the proposed contraceptive treatment. All patients answered the questionnaire before the application of LNG-IUS and six months after.

The purpose of the EHP-30 is to indicate the extent of self-reported ill health on each domain measured. Therefore, each scale is standardized on a scale of $0-100$, where 0 indicates the best health status through to 100 worst health status. Scale scores for each scale are calculated from the total of the raw scores of each item in the scale divided by the maximum possible raw score of all the items in the scale, multiplied by 100 . To measure the reliability of the instrument, the Cronbach's alpha (a coefficient of internal consistency) was used. Measures with reliability greater than 0.7 have been recommended as most useful in group-level hypothesis testing. To measure the temporal stability of the sample, the Intraclass Correlation Coefficient (ICC) was used. To estimate the magnitude of the sensitivity of the instrument to detect the change in quality of life among the two different times, the effect size was calculated by the difference between pre-treatment and post-treatment averages divided by the pre-treatment standard deviation, as recommended by Kazis et al. [12] A size of the effect equal to 0.20 indicates a small change, while measures of 0.50 and 0.80 indicate moderate and large changes respectively. A small effect size implies that treatment had little influence on the quality of life. To measure the difference between the means of each category Student t-test was used.

\section{Results}

The reliability of the EHP-30 was 0.99, the ICC for single measures was 0.73 ( $95 \%$ CI 0.66 to $0.84, p=0.000$ ) (Table 1). It proved to be sensitive to detect the effect size in the change of the responses. The comparative analysis of mean scores pre-post LNG-IUS application showed that the perception of quality of life improved significantly in all areas size effect of pain was $2.0(\mathrm{p}<0.0001)$; well-being and self-image $2.1(\mathrm{p}<0.0001)$; labor performance $0.6(\mathrm{p}<0.01)$; sexual intercourse $1.5(\mathrm{p}<0.0001)$ and overall treatment satisfaction $2.6(\mathrm{p}<$ 0.0001), respectively (Table 2).

Endometriosis is primarily associated with pain and infertility and has been postulated that the pain is responsible for causing the most negative effect on quality of life. LNG-IUS showed considerable improvement in the perception of pain with effect size of $2(\mathrm{p}<0.001)$ at the end of observation period.

Treatment tolerability: Adverse events were mild in nature and their incidences were intermenstrual spotting (13.8\%); headache (10.3\%); lower back pain, mastalgia and vulvar pruritus (3.4\%); 19 patients reported no adverse events during the study (65.5\%).

Two patients (6.9\%) required the use of concomitant therapy with non-steroidal analgesics for relief of pain. Only one patient prematurely discontinued the treatment because bias of selection (she wanted to seek pregnancy).

\section{Discussion}

The present study showed short term significant improvement of QoL in patients with pain associated with endometriosis, treated with LNG-IUS using Endometriosis Health Profile (EHP-30-30) questionnaire. The effect 
Table 1. Corrected item to total correlation and scale internal reliability consistency on the EHP-30.

\begin{tabular}{|c|c|}
\hline Scale item $(n=29)$ & Corrected item to total correlation \\
\hline \multicolumn{2}{|l|}{ Pain scale (alpha = 0.96) } \\
\hline P1. Unable to go to social events & 0.69 \\
\hline P2. Unable to do jobs at home & 0.56 \\
\hline P3. Found it difficult to stand & 0.66 \\
\hline P4. Difficult to sit & 0.61 \\
\hline P5. Found it difficult to walk & 0.66 \\
\hline P6. Lost appetite/unable to eat & 0.57 \\
\hline P7. Been unable to sleep & 0.59 \\
\hline P8. Had to go to bed/lie down & 0.67 \\
\hline P9. Unable to do things you want & 0.45 \\
\hline \multicolumn{2}{|l|}{ Emotional well-being scale (self-image) $($ alpha $=0.96)$} \\
\hline E1. Generally felt unwell & 0.49 \\
\hline E2. Frustrated as symptoms not getting better & 0.58 \\
\hline E3. Frustrated as not able to control symptoms & 0.63 \\
\hline E4. Felt symptoms ruling your life & 0.47 \\
\hline E5. Felt depressed & 0.52 \\
\hline E6. Felt weepy/tearful & 0.53 \\
\hline E7. Had mood swings & 0.62 \\
\hline E8. Felt bad or short-tempered & 0.57 \\
\hline E9. Felt unable to tell people how you feel & 0.54 \\
\hline E10. Felt alone & 0.7 \\
\hline E11. Appearance has been affected & 0.3 \\
\hline \multicolumn{2}{|l|}{ Work $($ alfa $=88)$} \\
\hline W1. Time off work & 0.67 \\
\hline W2. Unable to do duties at work & 0.58 \\
\hline W3. Embarrassed at work & 0.7 \\
\hline W4. Guilty taking time off work & 0.52 \\
\hline W5. Worried not able to do job & 0.56 \\
\hline \multicolumn{2}{|l|}{ Intercourse (alpha = 0.83) } \\
\hline INT1. Pain on intercourse & 0.29 \\
\hline INT2. Worried about intercourse & 0.08 \\
\hline INT3. Avoided intercourse & 0.06 \\
\hline INT4. Guilty about not wanting intercourse & 0.15 \\
\hline INT5. Frustrated cannot enjoy intercourse & 0.04 \\
\hline \multicolumn{2}{|l|}{ Treatment (alpha $=0.90)$} \\
\hline T1. Treatment not working & 0.4 \\
\hline T2. Difficulty coping with side effects & 0.35 \\
\hline T3. Dr not doing anything for you & 0.53 \\
\hline T4. Dr thinks it's in your mind & 0.27 \\
\hline T5. Dr lacks knowledge & 0.59 \\
\hline
\end{tabular}


Table 2. Effect size of change on EHP-30 parameters.

\begin{tabular}{|c|c|c|c|c|c|}
\hline & Basal & $6 \mathrm{mo}$ & Mean change score ${ }^{\&}$ & Effect size $^{*}$ & $\mathrm{p}$ \\
\hline Pain & $69.9 \pm 27.6$ & $12.8 \pm 17.4$ & 57.1 & 2 & 0.0001 \\
\hline Self-image & $68.3 \pm 26.5$ & $12.0 \pm 17.3$ & 56.3 & 2.1 & 0.0001 \\
\hline Work & $27.4+36.8$ & $6.0+14.5$ & 21.4 & 0.6 & 0.01 \\
\hline Sexual intercourse & $47.2+25.6$ & $7.6+12.9$ & 39.6 & 1.5 & 0.0001 \\
\hline Perception of treatment & $72.2 \pm 24.0$ & $10.0 \pm 15.1$ & 62.2 & 2.6 & 0.0001 \\
\hline
\end{tabular}

${ }^{*}$ A size of the effect equal to 0.20 indicates a small change, while measures of 0.50 and 0.80 indicate moderate and large changes respectively; ${ }^{\&}$ Mean score $(0-100) \pm$ s.d.

size magnitude in this study was higher with LNG-IUS treatment compared with the results obtained by Jones G. et al. [11], in women with only conservative surgery for the treatment of endometriosis-associated pain, precluding the necessity to develop further comparative studies.

In this study, the EPH-30 questionnaire showed high reliability in our population and a high sensitivity to detect the magnitude of change, demonstrating that treatment had clinically significant positive influence on our patients' health. The diagnosis of clinical suspicion by primary care physicians was low in this study (6.9\%), suggesting that provision of Continuing Medical Education should be done in order to improve the knowledge and awareness of this entity.

The limitations of this study are: a small number of subjects, the lack of a comparator (only 3 of 29 patients were not surgical treated) which is a strong bias to conclude any pure effect of LNG-IUS treatment of pain associated with endometriosis at least in the short term. Thus, it is recommend to perform comparative studies (LNGIUS vs. surgical treatment alone) with longer follow up to further determine the long lasting effect of LNG-IUS treatment in the control of endometriosis pelvic pain.

\section{Conclusion}

In this study, the LNG-IUS improved the quality of life of patients with endometriosis in all areas evaluated by EHP-30 including self-esteem, sexual intercourse, work and overall treatment satisfaction. We believe that LNG-IUS may represent an effective and convenient alternative in the long-term management of pelvic pain associated with endometriosis.

\section{Acknowledgements}

Authors are grateful with Dr. Leopoldo Alejandro Vazquez-Estrada for his assessment with the final manuscript.

\section{References}

[1] Schrager, S., Falleroni, J. and Edgoose, J. (2013) Evaluation and Treatment of Endometriosis. American Family Physician, 87, 107-113.

[2] Haas, D., Chvatal, R., Reichert, B., et al. (2012) Endometriosis: A Premenopausal Disease? Age Pattern in 42,079 Patients with Endometriosis. Archives of Gynecology and Obstetrics, 286, 667-70. http://dx.doi.org/10.1007/s00404-012-2361-z

[3] Hansen, K.A., Chalpe, A. and Eyster, K.M. (2010) Management of Endometriosis-Associated Pain. Clinical Obstetrics and Gynecology, 53, 439-448. http://dx.doi.org/10.1097/GRF.0b013e3181dbda06

[4] Balasch, J., Creus, M., Fábregues, F., et al. (1996) Visible and Non-Visible Endometriosis at Laparoscopy in Fertile and Infertile Women and in Patients with Chronic Pelvic Pain: A Prospective Study. Human Reproduction, 11, 387391. http://dx.doi.org/10.1093/HUMREP/11.2.387

[5] Preciado-Ruiz, R., Torres-Calleja, J., Zúñiga-Montiel, J., et al. (2005) Incidencia de la endometriosis en mujeres con infertilidad: Características clínicas y laparoscópicas. Ginecologia y Obstetricia de Mexico, 73, 471-476.

[6] Tekin, Y., Dilbaz, B., Kiykac Altinbas, S. and Dilbaz, S. (2010) Postoperative Medical Treatment of Chronic Pelvic Pain Related to Severe Endometriosis: Levonorgestrel-Releasing Intrauterine System versus Gonadotropin-Releasing Hormone Analogue. Fertility and Sterility, 95, 492-496. http://dx.doi.org/10.1016/j.fertnstert.2010.08.042

[7] Robinson, R., China, S., Bunkheila, A. and Powell, M. (2008) Mirena Intrauterine System in the Treatment of Men- 
strual Disorders: A Survey of UK Patients’ Experience, Acceptability and Satisfaction. Journal of Obstetrics and Gynaecology Research, 28, 728-731. http://dx.doi.org/10.1080/01443610802462605

[8] Centers for Disease Control and Prevention (2000) Measuring Healthy Days: Population Assessment of Health-Related Quality of Life. Centers for Disease Control and Prevention, Atlanta.

[9] Jones, G., Jenkinson, C. and Kennedy, S. (2004) The Impact of Endometriosis upon Quality of Life: A Qualitative Analysis. Journal of Psychosomatic Obstetrics and Gynaecology, 25, 123-133. http://dx.doi.org/10.1080/01674820400002279

[10] Jones, G., Kennedy, S., Barnard, A., Wong, J. and Jenkinson, C. (2001) Development of an Endometriosis Quality-ofLife Instrument: The Endometriosis Health Profile-30. Obstetrics \& Gynecology, 98, 258-264. http://dx.doi.org/10.1016/S0029-7844(01)01433-8

[11] Jones, G., Jenkinson, C. and Kennedy, S. (2004) Evaluating the Responsiveness of the Endometriosis Health Profile Questionnaire: The EHP-30-30. Quality of Life Research, 13, 705-713. http://dx.doi.org/10.1023/B:QURE.0000021316.79349.af

[12] Kazis, L.E., Anderson, J.J. and Meenan, R.F. (1989) Effect Sizes for Interpreting Changes in Health Status. Medical Care, 27, S178-S189. http://dx.doi.org/10.1097/00005650-198903001-00015

\section{Abbreviations}

LNG-IUS: Levonorgestrel-Releasing Intrauterine System;

QoL: Quality of Life;

EHP-30: Endometriosis Health Profile-30;

ASRM: American Society of Reproductive Medicine;

LUNA: Laparoscopic Uterine Nerve Ablation;

aGnRH: Analogues-Gonadotropin-Releasing Hormone Analogue;

HRQoL: Health Related Quality of Life;

PRO: Patient Self-Report of Outcomes;

ICC: Correlation Coefficient. 